\title{
Vehicular connectivity in urban scenarios: effectiveness and potential of roadside, moving WAVE providers and hybrid solutions
}

\author{
Claudia Campolo ${ }^{*}$, Hector Agustin Cozzetti ${ }^{2}$, Antonella Molinaro ${ }^{1}$ and Riccardo Scopigno ${ }^{2}$
}

\begin{abstract}
Vehicular ad-hoc networks are expected to be a key enabling technology for the development of future Intelligent Transportation Systems (ITSs), by delivering a wide range of services, spanning from safety alerting to route guidance and entertainment. Most of ITS applications require vehicles on the road to access the Internet through wireless communications with road-side units. The high vehicle mobility coupled with the large amount of investments required for deploying a complete roadside infrastructure will cause vehicle-to-roadside (V2R) connectivity to be poor, short-lived, and intermittent by negatively affecting the performance of envisioned applications. The purpose of this paper is to gain a deeper insight into possible issues related to service access and provisioning when considering the multi-channel operations envisioned by the IEEE $802.11 \mathrm{p} /$ WAVE (Wireless Access in Vehicular Environments) standards. We investigate network connectivity offered by roadside and moving WAVE providers in a realistic urban scenario where wireless propagation is hindered by obstructions. Results prove that hybrid solutions complementing roadside providers with moving ones lead to improved connectivity and data delivery performance, by potentially incurring lower deployment costs.
\end{abstract}

\section{Introduction}

Several ongoing research efforts supported by car and electronic industries, governments and academia are underway to foster the deployment of Vehicular Ad-Hoc NETworks (VANETs). VANETs are expected to be a key enabling technology for the development of Intelligent Transportation Systems (ITSs) meant to improve the quality of transportation by enabling a broad range of applications: primarily safety but also services aimed at traffic management, enhanced drive comfort, audio/ video streaming and generalized information and entertainment.

Vehicles may need to exchange with remote ITS servers a wide range of heterogeneous information ranging from environmental data (e.g., pollution measurements, average vehicle density and speed) to informative contents (e.g., e-maps, news items, proximity advertisements, etc).

\footnotetext{
* Correspondence: claudia.campolo@unirc.it

'DIMET-Dipartimento di Informatica, Matematica, Elettronica e Trasporti,

Università Mediterranea di Reggio Calabria, Reggio Calabria, Italy Full list of author information is available at the end of the article
}

(c) 2011 Campolo et al; licensee Springer. The paper is an extended version of the paper "Roadside and Moving WAVE Providers: Effectiveness and Potential of Hybrid Solutions in Urban Scenarios" presented at the 11th International Conference on

Telecommunications for Intelligent Transport Systems (ITST 2011). This is an Open Access article distributed under the terms of the Creative Commons Attribution License (http://creativecommons.org/licenses/by/2.0), which permits unrestricted use, distribution, and reproduction in any medium, provided the original work is properly cited.

To this purpose, roadside units (RSUs), located along the road or in points-of-interest, are needed to provide Internet access to on board units (OBUs) in vehicles. However, especially in the early deployment stages of VANETs, the coverage of RSUs will not be complete due to the high costs for planning, deploying, and maintaining an ubiquitous roadside infrastructure.

Poor, short-lived, and intermittent connectivity will be provided to users on vehicles by negatively affecting the overall performance of vehicular applications. Therefore, further connectivity solutions need to be explored: special vehicles (e.g., police cars, highway assistance or firefighting vehicles, public transportation means like buses or trams) and private cars could share their Internet access and act as gateways toward the Internet.

The purpose of this paper is to gain a deeper insight into the topic of vehicular connectivity in challenging urban scenarios by considering the features of the multi-channel architecture envisioned by the IEEE 802.11p [1] standard, recently ratified as an amendment of the IEEE 802.11 to provide Wireless Access in Vehicular Environments (WAVE) [2]. A single common control channel $(\mathrm{CCH})$ is used for safety messages and 
control frames delivery, while multiple service channels (SCHs) are for non-safety applications.

According to WAVE specifications, nodes offering connectivity services and the provisioning of non-safety applications over SCHs can be both RSUs and OBUs and they are called providers.

Connectivity offered by WAVE providers can heavily change depending on network and environmental conditions. Indeed, it is expected to be affected by positions of providers and their mobility patterns (in case of OBUs), street layouts, road congestion, fading and other propagation effects like diffractions, reflections and obstructions which are the aspects which mostly differentiate urban from other road settings.

Despite very complex, these phenomena have been recently demonstrated to be effectively simulated by simple (and not simplistic) models [3-5]. While the suggested models are straightforward, they adhere to and are validated by existing measurements $[4,6]$ and, what makes them relevant, can deeply influence and change the results.

This paper exploits some recent modeling achievements [3] to study the novel topic of WAVE connectivity offered by roadside and moving nodes in a urban setting. The model in [3] accounts for urban obstructions and has been integrated into the widely used network simulator NS-2 [7]. There it has been justified by measurements from literature and here is validated by means of ray-tracing software, confirming the reliability and accuracy of the additional attenuation values selected for urban simulations.

The main contributions of the paper can be summarized as follows:

- the investigation of connectivity performance in the realistically modeled urban scenario by considering different settings: RSUs differently placed on the road, OBUs providers, and hybrid solutions leveraging the coexistence of roadside and moving WAVE providers and by accounting for the specifications of the IEEE 802.11p/WAVE multi-channel architecture; - the performance evaluation of data delivery (e.g., ITS-related content) on service channels when only RSUs act as providers and when hybrid connectivity solutions are considered.

The rest of the paper is organized as follows. Section 2 describes the main features of IEEE 802.11p and WAVE; in Section 3, the obstruction model is presented and further validated by a ray-tracing software; simulation settings and results are respectively presented and discussed in Sections 4 and 5; conclusions are finally drawn in Section 6.

\section{WAVE protocol suite}

The IEEE $802.11 \mathrm{p}$ task group [1] has specified enhancements to the 802.11 physical (PHY) and medium access control (MAC) layers to address communications in vehicular environments.

The PHY layer is an amendment of 802.11a, and it is based on Orthogonal Frequency-Division Multiplexing (OFDM). Compared with IEEE 802.11a, the main differences concern time-parameters which get doubled in 802.11p to cope with the harsh vehicular environment: this is aimed at counteracting simultaneously inter-carrier interferences due to Doppler spread and inter-symbol interferences due to fading. As a result, halved data rate values are available in $802.11 \mathrm{p}$ ranging from 3 to 27 Mbps.

Seven $10 \mathrm{MHz}$-wide channels are available in the frequency band of 5.85-5.925 GHz allocated for the Dedicated Short Range Communication (DSRC)-based ITS services in US.

The European Telecommunications Standard Institute (ETSI) has also allocated a similar radio spectrum of 50 MHz. In both spectrum, one of the channels is reserved as a control channel for the exchange of system control and time-sensitive safety messages, while the rest (up to six in US and up to four in Europe) are service channels available to exchange not critical non-safety data packets, e.g. infotainment and ITS-related contents.

The IEEE 802.11p MAC layer exploits the Enhanced Distributed Channel Access (EDCA) scheme, which provides differentiated and distributed channel access.

IEEE 802.11p cooperates with the IEEE 1609 family [2], covering higher protocol layers, to define a standard protocol stack for vehicular environments.

Among the IEEE 1609 documents, the IEEE 1609.4 [8] enhances the 802.11p MAC layer to work in a multichannel environment. WAVE devices are expected to be deployed either as single-radio devices, which operate on one radio channel at a time or as dual-radio devices, which are capable of simultaneously monitoring control and service channels.

In a WAVE environment, both kinds of devices must have the possibility to tune in the same channel at the same time so that they can communicate. Therefore, above the IEEE 802.11p MAC layer, the 1609.4 specifications define four channel access switching modes: continuous, alternating, immediate, and extended access, Figure 1. According to the continuous access scheme, a node always stays tuned to the $\mathrm{CCH}$ to exchange safetyrelated data.

A node working in the alternating access scheme switches between the $\mathrm{CCH}$ and the available SCHs at scheduled time intervals. Specifically, the channel time is divided into synchronization intervals with a fixed 


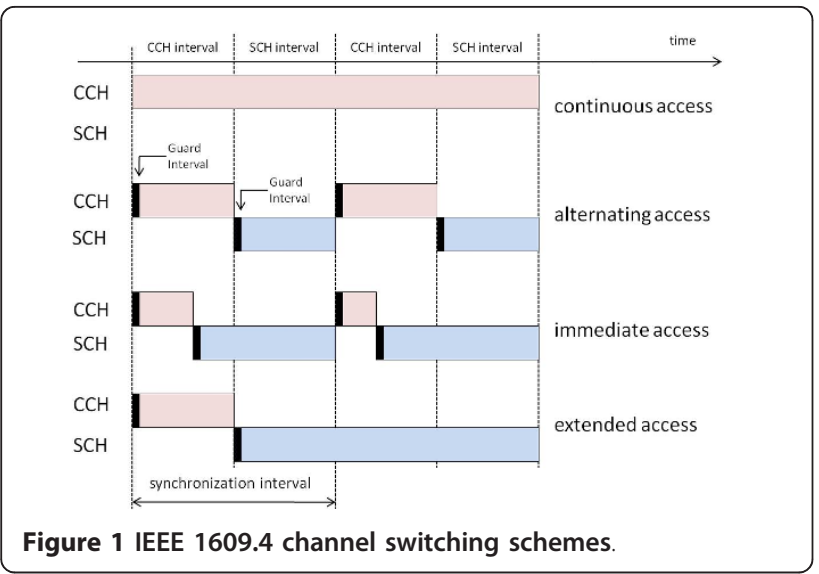

length of $100 \mathrm{~ms}$, consisting of $50 \mathrm{~ms}$-long $\mathrm{CCH}$ and $\mathrm{SCH}$ interval. Single-radio devices have to monitor the $\mathrm{CCH}$ during common time intervals (the $\mathrm{CCH}$ intervals), and to (optionally) switch to one $\mathrm{SCH}$ during the $\mathrm{SCH}$ intervals. Dual-radio devices can tune one radio into $\mathrm{CCH}$, while a second radio could be tuned into one of the SCHs. The described operation allows the safety warning messages to be transmitted on $\mathrm{CCH}$, while non-safety data applications may simultaneously run over SCHs.

The immediate access allows immediate communications over the $\mathrm{SCH}$ without waiting for the next $\mathrm{SCH}$ interval, by avoiding the latency of the residual $\mathrm{CCH}$ interval. The extended access allows communications over the $\mathrm{SCH}$ without pauses for $\mathrm{CCH}$ access and is useful for services which require a huge amount of data to be transferred and take several periods to be delivered.

The latter two schemes can be only beneficial to those vehicles which are not interested in cooperative safety applications; therefore, proper use cases need to be investigated for them.

At the early deployment stages of VANETs WAVE devices are expected to be low cost single-radio devices mainly enforcing the alternating switching scheme.

Coordination between channels exploits a global time reference, such as the Coordinated Universal Time (UTC), which can be supposed to be provided by the Global Positioning System (GPS).

Nodes aiming to initialize a Basic Service Set (BSS) among vehicles to exchange non-safety data over SCHs are called providers. Each provider announces itself and the set-up of its BSS by periodical WAVE Service Advertisement (WSA) messages broadcasted to nearby, 1-hopfar nodes, during the $\mathrm{CCH}$ interval. WSAs contain the information about the offered services and the network parameters necessary to join the advertised BSS (its identification, its $\mathrm{SCH}$, its EDCA parameter sets, configuration parameters needed to access the Internet, etc.).
Providers broadcast WSAs without any feedback on their successful reception; thus, the standard suggests that each provider sends more WSAs in the $\mathrm{CCH}$ interval for reliability purposes.

Providers should choose the least congested $\mathrm{SCH}$ for their BSS set up in order to reduce interference between nearby BSSs. However, how this is to be done is not specified in the standard specifications.

Nodes looking for available services, namely WAVE users, should monitor the $\mathrm{CCH}$ listening for WSAs to learn about the existence and the operational parameters of available BSSs. If they receive at least a WSA frame from a nearby provider, in order to join the BSS during the subsequent service channel interval they simply switch on the $\mathrm{SCH}$ frequency advertised in the WSA and start to exchange data with the provider.

\section{Urban scenario and its criticalities}

Nowadays, network simulators, like NS-2 [7], have sped up the analysis of various network scenarios, under several settings. These tools enable a pretty precise understanding of protocol mechanisms but, under certain circumstances, provide arguable results on performances: the reason lies in the resources available to precisely implement protocol entities and in the simplistic descriptions of physical and channel phenomena. As a result, simulations could lead to purely conceptual and qualitative description of the physical events, especially in urban scenarios where interactions between nodes and surrounding environment become fundamental and, nevertheless, neglected.

Important improvements have been recently proposed to increase the realism of simulations: the new features include mobility patterns matching real maps [9], detailed receiver models accounting for Viterbi errorrecovery [10] and obstruction models accounting for the presence of buildings [3-5] and for the impact of vehicles acting themselves as obstacles [11]. According to [10], the impact of obstructions appears to be the most relevant.

The solutions proposed in [3-5] share a common idea: additional attenuations are introduced based on mutual positions of nodes to account for propagation obstructions and residual propagation due to diffractions and scatters.

In this paper, we exploit the model proposed in [3], whose main building block is the off-line identification of mutual positions among vehicles and buildings within a given topology.

Basically, the model classifies positions into Line-ofSight (LoS), Near-Line-of-Sight (NLoS), and non-Line-ofSight (nLoS). LoS conditions apply to vehicles in the same road (e.g., vehicles $S$ and $A$ in Figure 2), NLoS to vehicles on the legs of a crossing not farther than a 


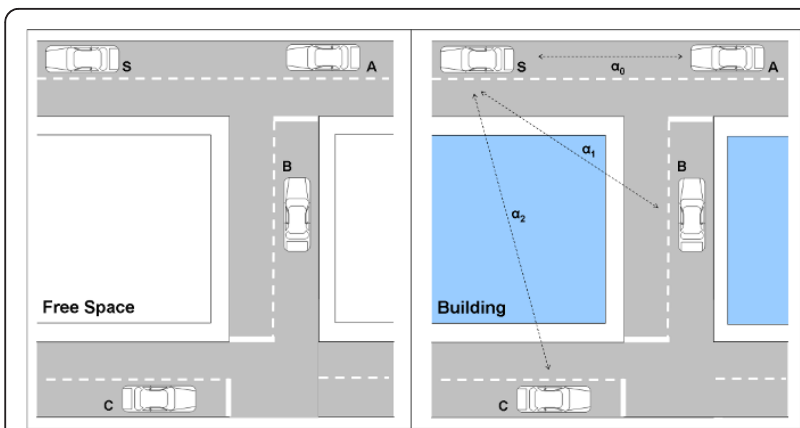

Figure 2 Zoom-in details of a crossroad area of the urban topology without and with buildings.

building (e.g., vehicles $S$ and $B$ in Figure 2), and nLOS to all the other cases (e.g., vehicles $S$ and $C$ in Figure 2).

This approach and the values of attenuation adopted to account for the presence of obstructions match the studies in [6,12], where real attenuation measurements are provided. For the sake of completeness, a further validation of this approach is here reported, based on simulations about propagation.

\section{Propagation model validation}

The urban attenuation model proposed in [3] is validated by means of a ray-tracing software, called Wireless InSite [13]. It is an advanced suite of ray-tracing models and 2D field solvers for the analysis of site-specific radio propagation and wireless communication systems. It provides efficient and accurate predictions of propagation and communication channel characteristics in complex urban, indoor, rural and mixed path environments.

A grid topology with the characteristics described in the next section, is simulated. The tool permits to select also materials, and the brick is selected as the main component of buildings. Half-wave dipole antennas with vertical polarization are configured in the frequency of $5.9 \mathrm{GHz}$ and $10 \mathrm{MHz}$ of channel bandwidth, compliantly with $802.11 \mathrm{p}$ specifications. The urban canyon propagation model is used to simulate the real environment. Ray-spacing, number of reflections and number of diffractions are some settable parameters that influence the power transferred from any active transmitter to all active receivers. Transmission power is set to $7 \mathrm{dBm}$.

Figure 3 highlights three transmitter positions and their rays that end on the reflection point on a building surface or terminate on the road boundary. In all these situations, the nodes in LoS with the source are able to correctly receive a packet, while the reception probability around the corner depends on mutual distances. Figure 4 shows the obstruction effect on received power at different distances. The crossroad causes a high drop of wireless signal, but not enough to completely prevent reception and, in the last case, half of the junction is still covered (the last reception takes place at about 60 meters from the center of the crossroad).

Altogether, these and other tests confirm that nodes along the same road are subject to a LoS propagation which leads to attenuation figures close to the Nakagami model, confirming results in [14]; nodes around a corner are subject to a propagation which is dominated by an extra-attenuation term and can be classified as NLoS; finally, the other cases fall in the category of nLoS and exclude reception by a heavy extra-attenuation term. These results confirm the attenuation values summarized in the next section, and derived from [3], for the deployed urban scenario.

\section{Simulation settings}

The performance analysis is carried out in a $750 \mathrm{~m}$-wide grid including $5 \times 5$ two-lane roads, spaced $150 \mathrm{~m}$ apart.

The mobility traces for 451 nodes moving along the simulated roads, with a mean speed of $60 \mathrm{~km} / \mathrm{h}$, are generated by SUMO [9]. Mobility traces feed NS-2 network simulator, in its enriched version [15] addressing an improved realism of the wireless channel with features such as: computation of cumulative Signal-toInterference and Noise Ratio (SINR) and the involvement of the modulation schemes in the decision on packet reception.

The signal strength of each received packet is computed by considering a statistical component modeling fading and a deterministic one accounting for urban obstructions. The statistical component follows the Nakagami distribution. In order to model medium fading conditions, the Nakagami fading intensity parameter, $m$, is set equal to 3 .

The additional component accounting for the obstructions follows the rules for extra-attenuation based on the classification of mutual positions of nodes with respect to obstacles (LoS, NLoS, nLoS) which have been derived in the model proposed in [3] and validated in the previous section. The following extra-attenuation parameters: $\alpha_{0}=0 \mathrm{~dB}, \alpha_{1}=-13 \mathrm{~dB}, \alpha_{2}=-30 \mathrm{~dB}$ have been used to recreate the effect of obstacles under LoS, NLoS, and nLoS conditions, respectively.

WAVE multi-channel operational mode and the alternating channel switching procedure at every $50 \mathrm{~ms}$ are built on the top of the PHY and MAC layers to simulate the behavior of single-radio devices. The data rate is set to $6 \mathrm{Mbps}$ and the transmission power is set to $7 \mathrm{dBm}^{\mathrm{a}}$. The main MAC parameters correspond to the common 802.11p configurations (e.g., slot-time $13 \mu \mathrm{s}$, SIFS time $32 \mu \mathrm{s}$, header length $40 \mu \mathrm{s}$, aCWmin 15 and aCWmax 1023). The size of WSA frames transmitted by providers is set to 200 bytes, while 7 is the retry limit value for data packets transmitted during the $\mathrm{SCH}$ interval. 


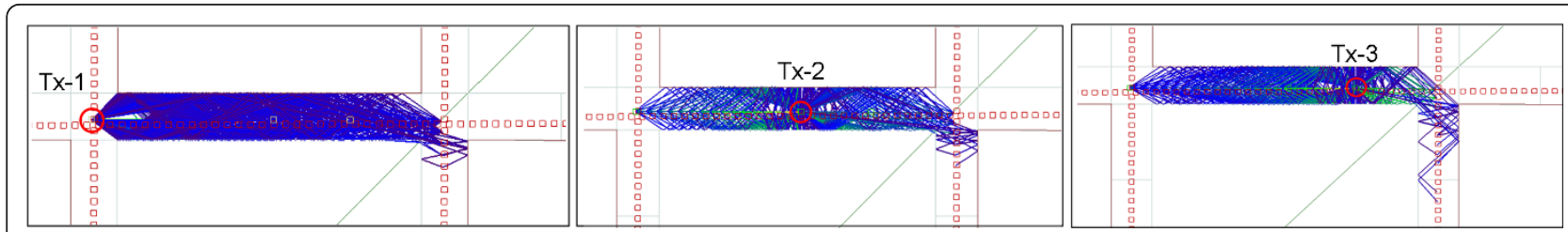

Figure 3 Ray-tracing patterns for three different positions of the transmitter and receivers in near-line-of-sight (around the corner).

\section{Analysis of results}

Fixed WAVE providers versus moving WAVE providers

The first set of results aims to evaluate the effectiveness of the BSS advertisement procedure when comparing the connectivity degree achieved by deploying either fixed (RSUs) or moving providers (OBUs).

The metric used to this purpose is the percentage of connected users, i.e., the percentage of vehicles that have received (at least) one WSA frame from a nearby provider during the $\mathrm{CCH}$ interval, and, hence, have the possibility to exchange data with it during the successive $\mathrm{SCH}$ interval. The achieved percentage values are mean values averaged throughout several simulation runs.

Different placements of the RSUs in the studied topology are considered, Figures 5, 6, and 7. Curves labeled as scenario (a) in Figures 8, 9, and 10 respectively refer to the percentage of connected users for 6,10 , and 14 RSUs, deployed in strategic places, i.e., the intersections, as depicted in Figure 5.

Another proposed scenario (depicted in Figure 6) is made up of 6,10 and 14 fixed roadside units placed only at the sides of the roads. In this configuration, there are not any RSUs situated nearby the crossroads, restricting the wireless communication coverage. The simulation results achieved by these configurations are

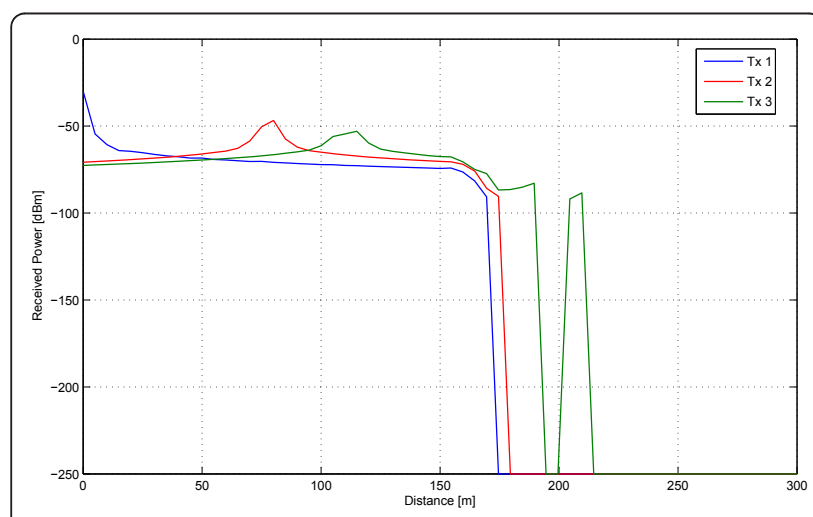

Figure 4 Received power as a function of distance in the urban scenario under study: the three graphs correspond to the possible positions of transmitter; distances are Euclidean and not cartesian and measured along a path starting at the transmitter and turning the corner. The sharp falls correspond to corner turning. presented in Figures 8, 9 and 10 respectively (curves labeled as scenario (b)).

Finally, a hybrid scenario is used to cover the case when RSUs (6,10 and 14 nodes) are placed both in the crossroads and along the streets, Figure 7. Curves labeled as scenario (c) in Figures 8, 9 and 10 highlight their corresponding results.

For the sake of completeness, in all these cases, the infrastructure nodes are homogeneously distributed in the urban area: their location is determined by the transmitted power based on the involved urban distances. In fact, RSUs placement is always planned to cover areas as wide as possible, also considering effects of urban obstructions.

Curves labeled as OBUs shows the same metric when a variable number of moving providers $(6,10$, and 14) are randomly selected among the 451 deployed vehicles.

As a general result, it can be observed that by increasing the number of WSA repeats, a higher percentage of

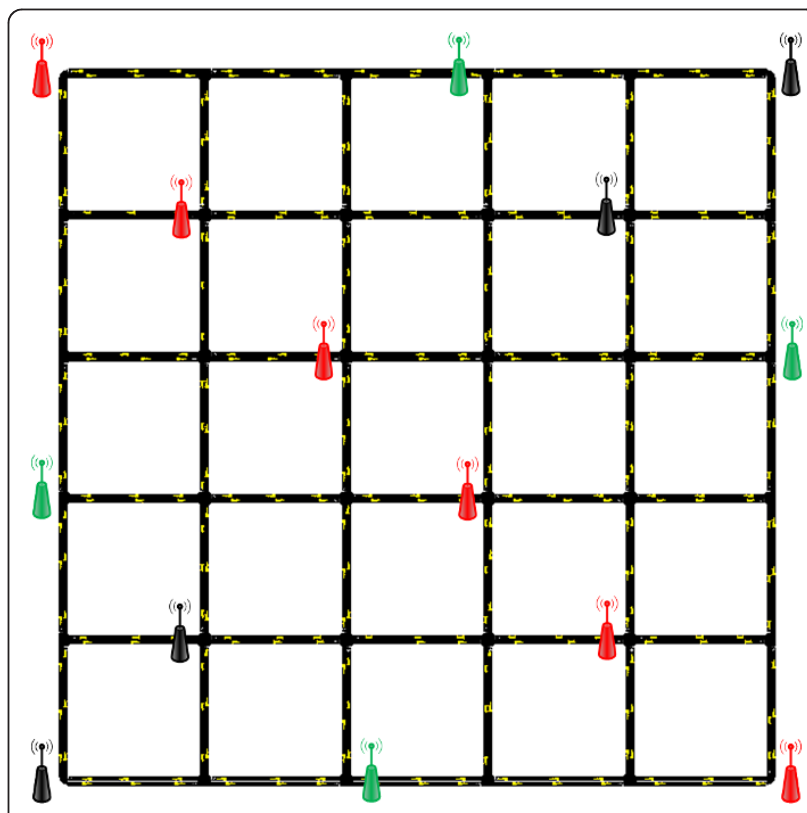

Figure 5 RSUs positions in the Manhattan-like grid topology: scenario (a). The three simulated cases are distinguished by colors: 6-RSUs displayed as red units; 10-RSUs: red + black; 14-RSUs: red + black + green 


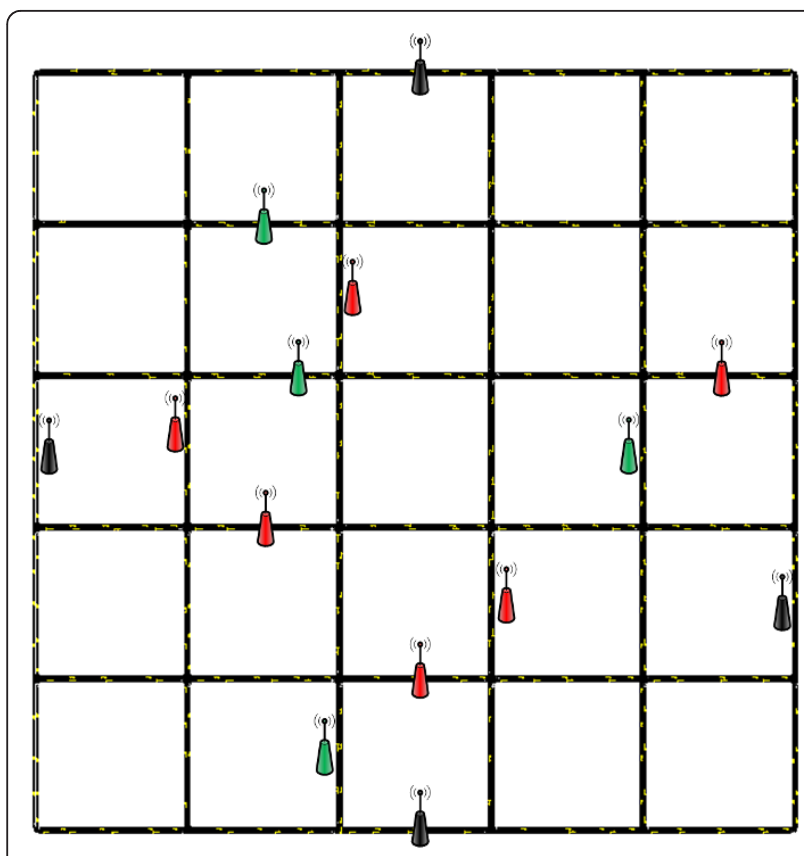

Figure 6 RSUs positions in the Manhattan-like grid topology: scenario (b). The three simulated cases are distinguished by colors: 6-RSUs displayed as red units; 10-RSUs: red + black; 14-RSUs: red + black + green

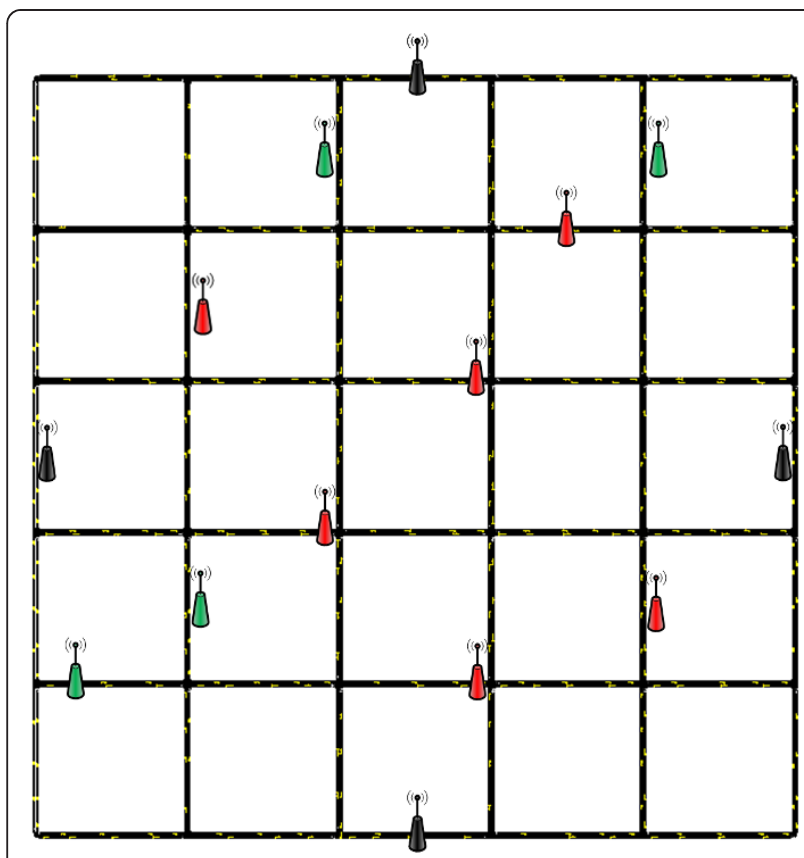

Figure 7 RSUs positions in the Manhattan-like grid topology: scenario (c). The three simulated cases are distinguished by colors: 6-RSUs displayed as red units; 10-RSUs: red + black; 14-RSUs: red + black + green

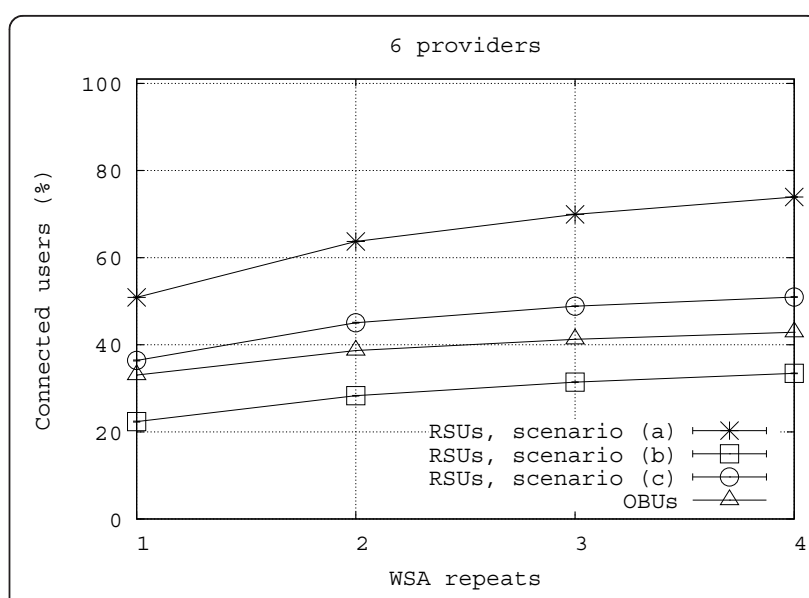

Figure 8 Percentage of connected users versus WSA repeats when 6 providers are considered in different scenarios.

connected users is achieved in all cases since, by repeating WSAs, losses due to fading are counteracted.

The best connectivity performance is achieved when considering scenario (a) with RSUs at the intersections. The presence of buildings instead dramatically affects the connectivity in all other cases.

With 10 RSUs positioned at intersections, almost full connectivity is achieved when setting the WSA repeats to 4 , i.e., almost each vehicle is under the coverage of at least one provider. Conversely, by fixing the same number of OBUs as WAVE providers only $60 \%$ of users are connected.

The worst connectivity performance are achieved when RSUs are positioned along the street, scenario (b). This is because WSA transmissions of a RSU provider along a street is mainly confined along the same street, with a limited propagation around obstructed corners.

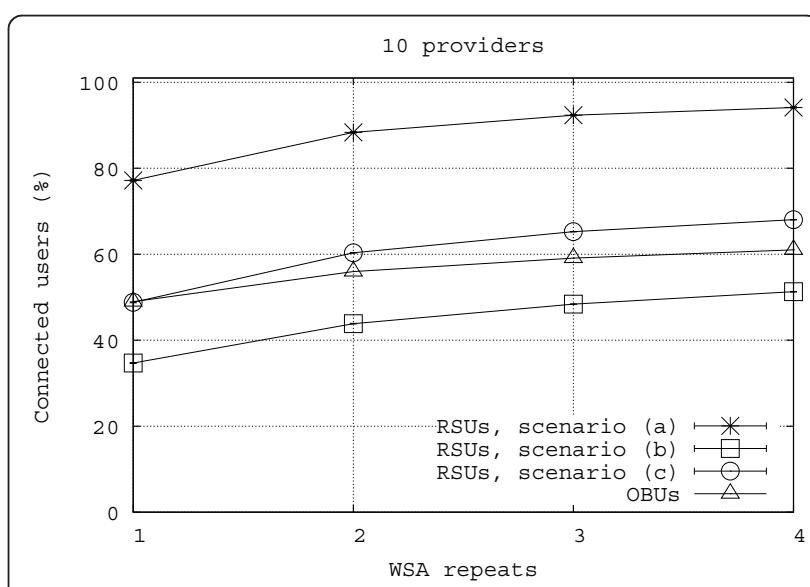

Figure 9 Percentage of connected users versus WSA repeats when 10 providers are considered in different scenarios. 


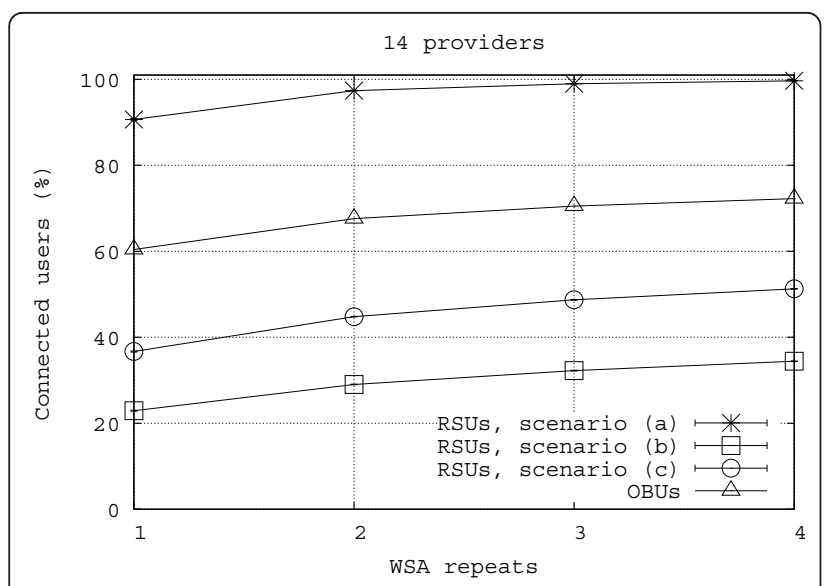

Figure 10 Percentage of connected users versus WSA repeats when 14 providers are considered in different scenarios.

RSUs deployment as in Scenario (c) partially solves this issue by increasing connectivity.

The higher the number of providers, the better the connectivity, with the exception of 14 RSUs positioned as in Scenario (b) and Scenario (c). In such a case, WSAs from nearby RSUs could experience collisions, by hindering vehicles to detect a nearby RSU.

The main finding suggested by the achieved results is that position of RSUs plays a crucial role in determining the connectivity performance when realistic propagation conditions are considered in urban scenarios.

\section{Hybrid scenario: fixed and moving providers}

The previous results show that it is preferable to rely on a roadside infrastructure in order to provide connectivity to vehicles. However, given the required elaborate and proper placement design and the seemingly large cost of ubiquitous network infrastructure along the road, in terms of power and wired network connectivity, only a few RSUs will be likely installed in the near future.

On the other hand, a solution where all the providers are moving -i.e., the backbone is made up only by special vehicles (police cars, buses, trams) offering connectivity services to nearby vehicles- would guarantee an easy, low-cost, fast, and low power deployment, but at the expenses of a scarce connectivity, unless a very high number of moving providers is involved (50 vehicles in our tests on the considered urban scenario).

Considering this foreground, it seems critical to investigate a hybrid scenario where both RSUs and OBUs act as WAVE providers: this is covered by the simulations presented in this subsection. Connectivity provided by six RSUs positioned as in Figure 5 is complemented by a variable number of moving providers randomly selected among vehicles in the grid.
To evaluate the effectiveness of a hybrid connectivity solution in supporting the delivery of non-safety applications, we consider a variable number of vehicles (from 50 to 200) transmitting a 1000-byte unicast data frame to a detected provider during each $\mathrm{SCH}$ interval with priority set equal to best effort [1].

The simulated traffic generation pattern could resemble the case of vehicles uploading information about the nearby environment, e.g., detected through their on board sensors and cameras, to remote servers accessed through providers.

Some nodes may be able to detect more providers, either RSUs and OBUs, during a given $\mathrm{CCH}$ interval. In such a case, vehicles always join the nearest BSS, i.e., they switch on the $\mathrm{SCH}$ advertised by the nearest provider. The assumption of awareness about the proximity of a provider is reasonable since (i) each vehicle is supposed to be equipped with a positioning system -hence to know about its current location- and (ii) every provider includes its own position in the LATITUDE and LONGITUDE fields of the WSA frame, as suggested by the standard.

The metrics used to evaluate the performance of data exchange during the $\mathrm{SCH}$ interval are packet delivery ratio (PDR) and delay. PDR accounts for the percentage of packets successfully delivered to the provider; packet delay accounts for the latency accumulated by a packet from its generation time, at the user side, to the delivery at the target provider.

In order to achieve a fair comparison, evaluation is conducted in the two following scenarios: (i) only 10 RSUs positioned at intersections, and (ii) 6 RSUs plus additional 30 OBUs providers are deployed, which offer similar connectivity performance, i.e., nearly $98 \%$ of connected users, as depicted in Figure 11.

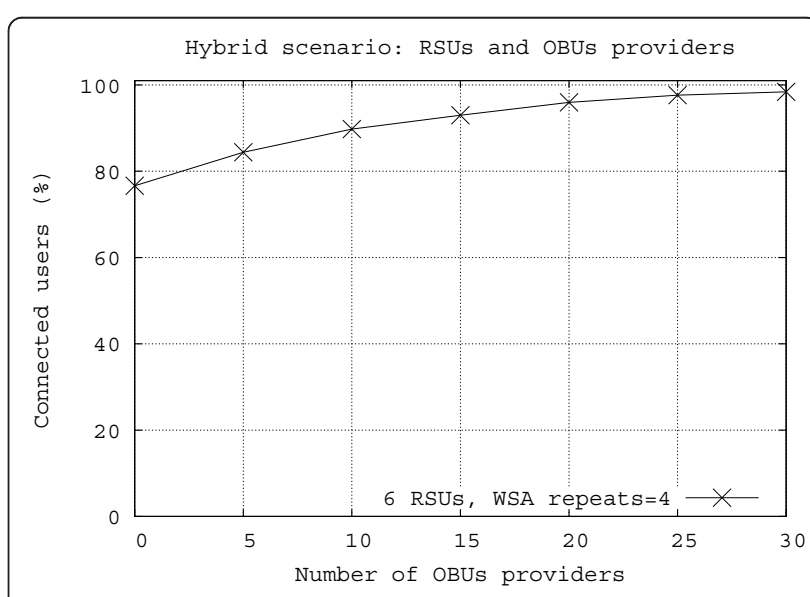

Figure 11 Percentage of connected users in the hybrid scenario when varying the number of OBUs providers. 


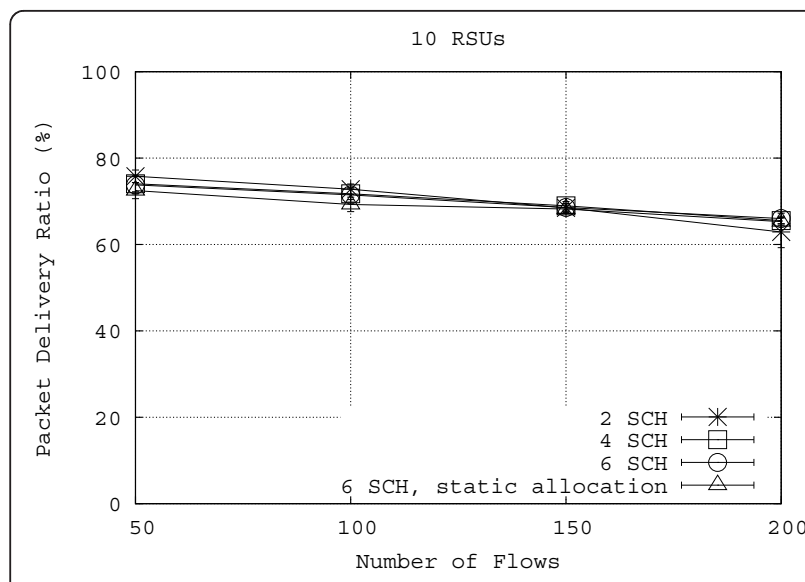

Figure 12 Packet delivery ratio for data transmissions on the SCH interval when 10 RSUs are deployed in the scenario when varying the number of $\mathrm{SCHs}$.

PDR and delay results for scenario (i) and (ii) are respectively shown in Figures 12 and 13, 14 and 15.

The number of SCHs is variable in our study $(2,4,6)$ to reflect the current trends in the ETSI and DSRC context. Moreover, the variation of this parameter allows a better understanding of the impact of intra-BSS and inter-BSS interference, respectively referring to the amount of collisions and interference among nodes belonging to the same BSS and nodes belonging to different but spatially overlapping BSSs, working on the same service channel.

In order to reduce interference between different BSSs, we let providers striving to initialize them on different SCHs. A simple policy has been deployed ${ }^{\mathrm{b}}$, according to which, during the $\mathrm{CCH}$ interval, each provider monitors the status of the service channels by receiving WSAs and chooses as $\mathrm{SCH}$ for its $\mathrm{BSS}$ one of

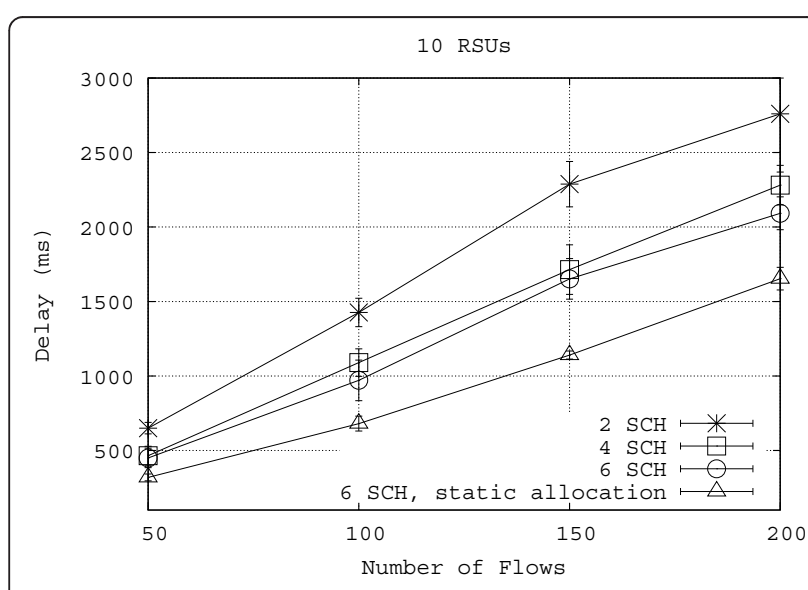

Figure 13 Delay for data transmissions on the $\mathrm{SCH}$ interval when 10 RSUs are deployed in the scenario when varying the number of $\mathrm{SCHs}$

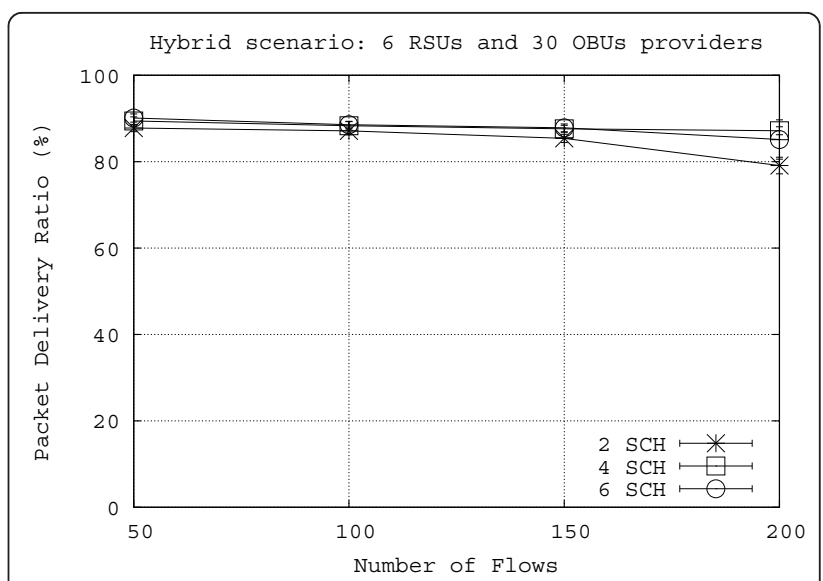

Figure 14 Packet delivery ratio for data transmissions on the $\mathrm{SCH}$ interval in the hybrid scenario when varying the number of SCHs.

the $\mathrm{SCH}$ perceived as free. If all $\mathrm{SCH}$ are reserved, a provider randomly chooses an $\mathrm{SCH}$ among the busy ones.

Additionally, a static $\mathrm{SCH}$ allocation policy is foreseen for the scenario with only RSUs acting as WAVE providers. In fact, since they are statically placed and they can be connected by a wired infrastructure, service channels can be pre-allocated to RSUs in order to avoid their BSSs to be channel-overlapping ${ }^{\mathrm{c}}$.

As expected, by looking at Figures 12, 13, 14 and 15, it can be observed that performances get worse when increasing the number of active flows, with highly increasing delay values and decreasing PDR trends.

The same effect in terms of delay is observed when reducing the number of $\mathrm{SCH}$. This is because pairs of user/provider in the same radio coverage and simultaneously communicating in channel-overlapping BSSs could interfere. As a consequence, the higher contention

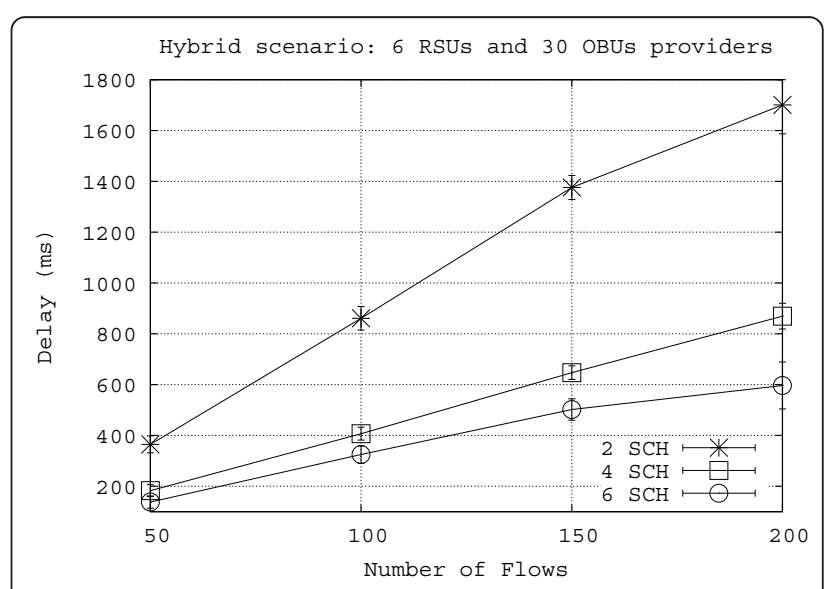

Figure 15 Delay for data transmissions on the $\mathrm{SCH}$ interval in the hybrid scenario when varying the number of SCHs. 
on the channel and retransmissions triggered by packet losses due to collisions and interference increase the delay. Such a worsening in delay performance is especially noticed when passing from $4 \mathrm{SCHs}$ to $2 \mathrm{SCHs}$, since it is more likely that nearby BSSs are initialized to work on the same $\mathrm{SCH}$ when only $2 \mathrm{SCH}$ are available in the channel spectrum.

Packet delivery ratio is not affected by the number of available service channels since packet losses can be recovered thanks to the enforcement of retransmission procedures.

Whatever the number of available $\mathrm{SCHs}$ and the traffic load, the hybrid connectivity solution always achieves better performance compared with the scenario when only RSUs act as providers.

When the static SCH allocation policy is enforced (curves labeled as static allocation), which allows the allocation of service channels in such a way to nullify the inter-BSS interference, worse performances are achieved as compared to the hybrid scenario as well.

The main reason behind such results is that a higher number of nodes (OBUs) acting as WAVE providers allow vehicles to better distribute among the available BSSs, as shown in Table 1. The first row accounts for the average number of users per BSS when only 10 RSUs are considered. The second and the third line respectively refer to the average number of users joining the BSS of one of the 6 available RSUs and the BSS of one of the 30 OBUs acting as WAVE providers in the hybrid scenario. It is clear that a hybrid coverage guarantees a lower load per BSS, which is more than halved as compared to the scenario with 10 fixed providers. The achieved better load balancing leads to lower intraBSS interference.

Such a trend suggests that in the considered scenarios intra-BSS interference plays a more significant role on the performance as compared to the inter-BSS interference. This is due to the presence of obstructions that heavily confines signal propagation along the same street. Therefore, multiple simultaneous transmissions over the same $\mathrm{SCH}$ on different BSSs do not interfere if they are sufficiently apart (e.g., in parallel streets).

The main finding of the study reported in this subsection is that a hybrid connectivity solution, by incurring significantly lower deployments costs, has the further benefit to improve data delivery performance, thanks to

Table 1 Number of users per BSS, 4 WSA repeats

\begin{tabular}{lc}
\hline Providers & Users per BSS \\
\hline 10 RSUs & 42.45 \\
6 RSUs (hybrid scenario) & 14.7 \\
30 OBUs (hybrid scenario) & 10.63 \\
\hline
\end{tabular}

the better distribution of users among the existing providers.

\section{Conclusions}

The paper has presented a study aimed to evaluate the performance of the IEEE 802.11p/WAVE multichannel operation under novel and still unexplored settings. The analysis has been conducted in a challenging urban scenario characterized by the presence of obstructions and where WAVE services are provided alternatively by RSUs, by vehicles, and by both RSUs and vehicles.

Achieved results suggest that RSUs, if properly deployed in the streets (e.g., at intersections), can provide good connectivity to vehicles passing by, but may lead to high deployment costs. Moreover, a clear indication of the scarce connectivity provided by moving providers only in a urban scenario is also given.

Therefore, a solution addressing the trade-off between connectivity and easiness of deployment has been investigated: it leverages on the complementary involvement of roadside providers with moving ones.

Results show that the proposed solution, thanks to the achieved better load balancing of WAVE users among existing providers, has the additional benefit of improving the delivery performance of non-safety data exchanged on service channels.

\section{Endnotes}

${ }^{\mathrm{a}}$ With this value of transmission power, the maximum distance at which packet receptions are still possible by assuming a deterministic path loss component is $150 \mathrm{~m}$.

${ }^{\mathrm{b}}$ Different policies can be foreseen, as for example the one proposed in [16], however, the service channel selection issue is outside the main objective of this paper.

${ }^{c}$ Such an option is not viable for OBUs providers due the dynamicity of their route.

\section{Acknowledgements}

The authors would like to thank Dr. Riccardo Maggiora (Politecnico di Torino) for his kind support and the provision of ray-tracing results used within the urban propagation model.

\section{Author details}

${ }^{1}$ DIMET-Dipartimento di Informatica, Matematica, Elettronica e Trasporti, Università Mediterranea di Reggio Calabria, Reggio Calabria, Italy ${ }^{2}$ BWA Lab (Broadband Wireless Access), Istituto Superiore Mario Boella, Turin, Italy

\section{Competing interests}

The authors declare that they have no competing interests.

Received: 20 July 2011 Accepted: 28 October 2011

Published: 28 October 2011

\section{References}

1. IEEE Std. 802.11p, Wireless Access in Vehicular Environments (July 2010)

2. YL Morgan, Notes on DSRC \& WAVE standards suite: its architecture, design and characteristics. IEEE Commun Surv Tutor. 12(4), 1-15 (2010) 
3. R Scopigno, HA Cozzetti, in Proceedings of the ICWMC. Signal Shadowing in Simulation of Urban Vehicular Communications (2010)

4. E Giordano, R Frank, G Pau, M Gerla, CORNER: A Realistic Urban Propagation Model for VANET, in Proceedings of the 2010 Seventh International Conference on Wireless On-demand Network Systems and Services (WONS) (2010)

5. C Sommer, D Eckhoff, R German, F Dressler, A Computationally Inexpensive Empirical Model of IEEE 802.11p Radio Shadowing in Urban Environments, in WONS. (2011)

6. CohdaWireless Mobile Broadband, DSRC Field Trials. http://www. cohdawireless.com (June 2010)

7. NS-2, Network Simulator tool, http://www.isi.edu/nsnam/ns

8. IEEE 1609.4, IEEE Standard for Wireless Access in Vehicular Environments (WAVE)-Multi-channel Operation (February 2011)

9. SUMO, sub-urban mobility simulator. http://sumo.sourceforge.net

10. HA Cozzetti, et al, Improving Wireless Simulation Chain: Impact of Two Corrective Models for Vanets, in Proceedings of IEEE VNC. (2010)

11. M Boban, et al, Impact of vehicles as obstacles in vehicular ad hoc networks. IEEE J Sel Areas Commun. 29(1), 15-28 (2011)

12. P Alexander, D Haley, A Grant, Outdoor mobile broadband access with 802.11. IEEE Commun Mag. 45(11), 108-114 (2007)

13. Wireless EM, Propagation Software-Remcom Wireless InSite. http://www remcom.com/wireless-insite

14. V Taliwal, et al, Empirical Determination of Channel Characteristics for DSRC Vehicle-to-vehicle Communication. ACM VANET. (2004)

15. Q Chen, et al, Overhaul of IEEE 802.11 modeling and Simulation in NS-2. in Proceedings of ACM MSWiM. (2007)

16. C Campolo, A Cortese, A Molinaro, CRaSCH: A Cooperative Scheme for Service Channel Reservation in 802.11 p/WAVE Vehicular Ad Hoc Networks, in Proceedings of Nets4Cars, International Workshop on Communication Technologies for Vehicles. (2009)

doi:10.1186/1687-1499-2011-146

Cite this article as: Campolo et al:: Vehicular connectivity in urban scenarios: effectiveness and potential of roadside, moving WAVE providers and hybrid solutions. EURASIP Journal on Wireless

Communications and Networking 2011 2011:146.

\section{Submit your manuscript to a SpringerOpen ${ }^{\circ}$ journal and benefit from:}

- Convenient online submission

- Rigorous peer review

- Immediate publication on acceptance

- Open access: articles freely available online

- High visibility within the field

- Retaining the copyright to your article

Submit your next manuscript at $\gg$ springeropen.com 